\title{
¿Influyen las características psicológicas y los estilos de aprendizaje en el rendimiento académico de los estudiantes de medicina? Un estudio retrospectivo
}

\author{
Marcela Bitran $C^{a}$, Montserrat Lafuente $G$, \\ Denisse Zúñiga $\mathbf{P}^{b}$, Paola Viviani $G^{c}$, Beltrán Mena $C$. \\ The influence of phychological features \\ and learning styles on the academic \\ performance of medical students
}

Background: The degree of difficulty we experience while learning different concepts and skills depends, among other things, on our psychological features and learning style. This may be particularly true for medical students, whose formation involves the acquisition of multiple cognitive, affective and psychomotor skills. Aim: To assess whether the psychological features and learning styles of medical students are associated with their academic performance. Subjects and Methods: The psychological preferences and learning styles of 66 students of the 2001-graduating cohort were determined with the Myers Briggs Type Inventory (MBTI) and the Kolb Learning Style Inventory (LSI), respectively. The academic performance was assessed by the Calificación Médica Nacional (CMN), Chile and by the marks obtained during the Basic (1st to 3th), Preclinical (4th y 5th) and Clinical (6th y 7th) years of undergraduate training. Results: The psychological features, together with the sex of students were found to be associated with the performance in the Preclinical and Clinical years, and to the CMN. In men, the interest and ability to communicate with people and the concern for harmony, and in women the tendency to function in a systematic and orderly way are the features associated to high academic performance. No associations were found between learning styles and academic performance. Conclusions: The finding that the psychological preferences of medical students are relevant to their academic performance opens a new perspective to analyze the medical education and to design programs aimed at improving learning (Rev Méd Chile 2004; 132: 1127-36).

(Key Words: Education, medical; Learning; Students)

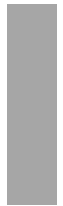

\footnotetext{
Recibido el 8 de enero, 2004. Aceptado en versión corregida el 12 de julio, 2004.

Trabajo realizado como parte del proyecto MECESUP de pregrado, PUC 008-2000.

Centro de Educación Médica, Escuela de Medicina, Pontificia Universidad Católica de Chile.

aDoctora en Farmacología

bPsicóloga

cMagíster en Estadísticas
}

Correspondencia a: Marcela Bitran C. Centro de Educación

Médica, Escuela de Medicina, Pontificia Universidad Católica de Chile. Alameda 340, edificio Pregrado Medicina 6o piso. Fono: 354-3811. Fax: 633-1457. E mail: mbitran@med.puc.cl 
L a forma en que percibimos y procesamos la información, y la dificultad que experimentamos al adquirir distintos conocimientos y habilidades están influidos por nuestra personalidad y estilo de aprendizaje. Estas variables han sido objeto de estudio, durante las últimas décadas, por parte de escuelas de medicina europeas y norteamericanas por su posible relación con el desempeño académico, la elección de especialidad y la satisfacción de sus alumnos ${ }^{1-8}$. En Chile se ha investigado la relación entre rasgos psicopatológicos y rendimiento académico en estudiantes universitarios $^{9,10}$, pero no hay estudios que relacionen características de personalidad de individuos clínicamente normales y desempeño académico.

En 2000, iniciamos en la Escuela de Medicina de la Pontificia Universidad Católica de Chile (PUC) un estudio longitudinal de 9 años de duración, llamado Psimed 21, destinado a evaluar si las características normales de personalidad y los estilos de aprendizaje de nuestros estudiantes se asocian de manera consistente con su desempeño en las distintas asignaturas o ciclos de la carrera $^{11}$

En una aproximación complementaria al Psimed 21, realizamos el presente estudio de carácter retrospectivo, destinado a indagar la relación entre las características de personalidad y los estilos de aprendizaje de los estudiantes que egresan, y su desempeño académico en los distintos ciclos de la carrera. Pensamos que, de existir, esta relación debiera ser más notoria en los ciclos preclínico y clínico, en los que intervienen de manera más importante las habilidades interpersonales.

Tomando en cuenta las limitaciones inherentes a su carácter retrospectivo, podremos utilizar los resultados de este estudio para pesquisar las tendencias más salientes que relacionan personalidad y estilos de aprendizaje con rendimiento y, a partir de ellas, guiar el desarrollo y enriquecer el análisis de los resultados que comienza a arrojar el estudio longitudinal (Psimed 21) iniciado el año 2000.

\section{MATERIALES Y MÉTODO}

Población. Participaron en este estudio 66 de los 78 estudiantes de la cohorte de egreso 2001 de la
Escuela de Medicina de la Pontificia Universidad Católica de Chile. El perfil biodemográfico y académico de estos estudiantes se detalla en la Tabla 1.

Variables psicológicas. Las características de personalidad se operacionalizaron a través de las preferencias psicológicas del MBTI (forma G, versión en español) ${ }^{12,13}$ y los estilos de aprendizaje según la clasificación de Kolb (LSI, versión en español) ${ }^{14}$. Los tests fueron aplicados a fines del primer semestre de 2001, en el $7^{0}$ año de la carrera, y previo consentimiento informado de los participantes.

El MBTI es un instrumento de autorreporte, válido y confiable ${ }^{13,16,17}$, ampliamente usado en educación médica ${ }^{8,18-20}$. Se desarrolló en base a la Teoría de Tipos Psicológicos de Carl Jung ${ }^{21}$ y, a diferencia de otros instrumentos, no mide rasgos de personalidad sino preferencias psicológicas. La preferencia psicológica se define como la inclinación natural de una persona a actuar según uno $u$ otro polo de 4 dimensiones psicológicas independientes $^{22}:$ 1) la fuente de motivación, 2) el modo de percibir la información, 3) el modo de discernir y 4) la actitud frente al mundo (Figura 1).

Los polos opuestos de la primera dimensión son Extraversion (E) e Introversion (I). Para un individuo con preferencia $\mathrm{E}$, la fuente de motivación está en el mundo externo, en contacto con las cosas y personas; si su preferencia es I, su motivación se originará principalmente de su mundo interior. Según su percepción de la información, las personas con preferencia Sensing (S) prestan

Tabla 1. Características de los estudiantes

\begin{tabular}{|ll}
\hline Antecedentes Biodemográficos & \\
Edad* & $25,2 \pm 1,2$ años \\
Mujeres & $39 \%$ \\
Primogénitos & $59 \%$ \\
No hermanos & $2,6 \pm 1,4$ \\
(incluido el egresado) & \\
Antecedentes académicos & \\
Puntaje de selección & $753 \pm 19$ puntos \\
Vía de ingreso regular & $95 \%$ \\
Colegio particular & $85 \%$ \\
Región metropolitana & $76 \%$
\end{tabular}

*Edad al egreso 


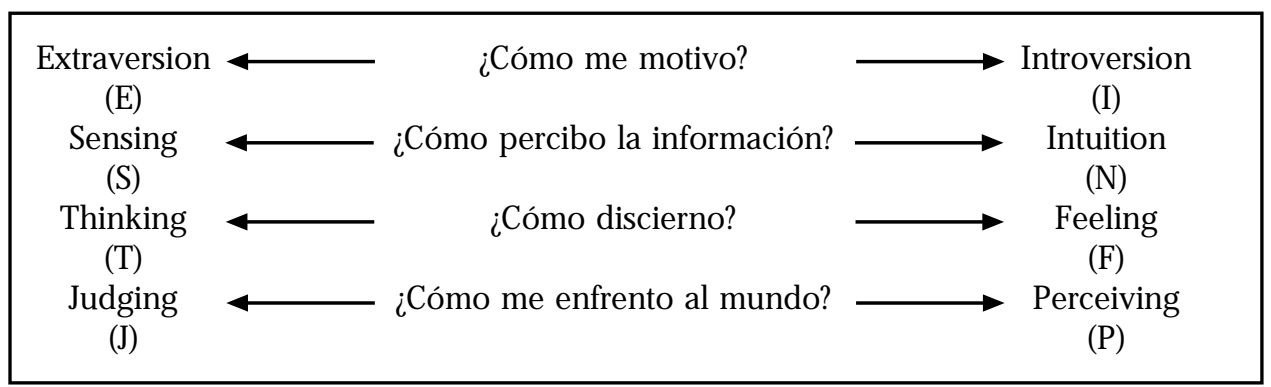

FiguRA 1. Preferencias psicológicas según el MBTI.

especial atención a los detalles y aspectos concretos; las con preferencia Intuition $(\mathrm{N})$ a los patrones generales y las posibilidades. En cuanto a su discernimiento, las personas con preferencia Thinking $(\mathrm{T})$ basarán sus decisiones en el análisis lógico e imparcial y las con preferencia Feeling $(F)$ en las convicciones y valores personales. Los polos de la cuarta dimensión Judging (J) y Perceiving (P), describen la actitud de las personas frente al mundo. La tendencia a vivir en forma planificada y a concretar decisiones rápidamente caracteriza a las personas con preferencia J. En contraste, las personas con preferencia $P$ tienen una disposición más perceptiva y flexible y menor urgencia por decidir.
El LSI identifica cuatro tipos de aprendices según su modo de adquirir y utilizar la información ${ }^{15}$, a saber, Asimilador, Convergente, Divergente y Acomodador (Figura 2). El Asimilador (abstracto-reflexivo) tiende a aprender sistematizando la información en teorías unificadoras o patrones y reflexiona acerca de ellos sin mucho interés en su aplicación práctica. El Convergente (abstracto-activo), aprende al aplicar el conocimiento a problemas luego de generar modelos hipotéticos. El Divergente (concreto-reflexivo), tiene facilidad para aprender de la experiencia una vez que la ha considerado desde múltiples perspectivas y el Acomodador (concreto-activo),
FIgURA 2. Estilos de aprendizaje según Kolb.

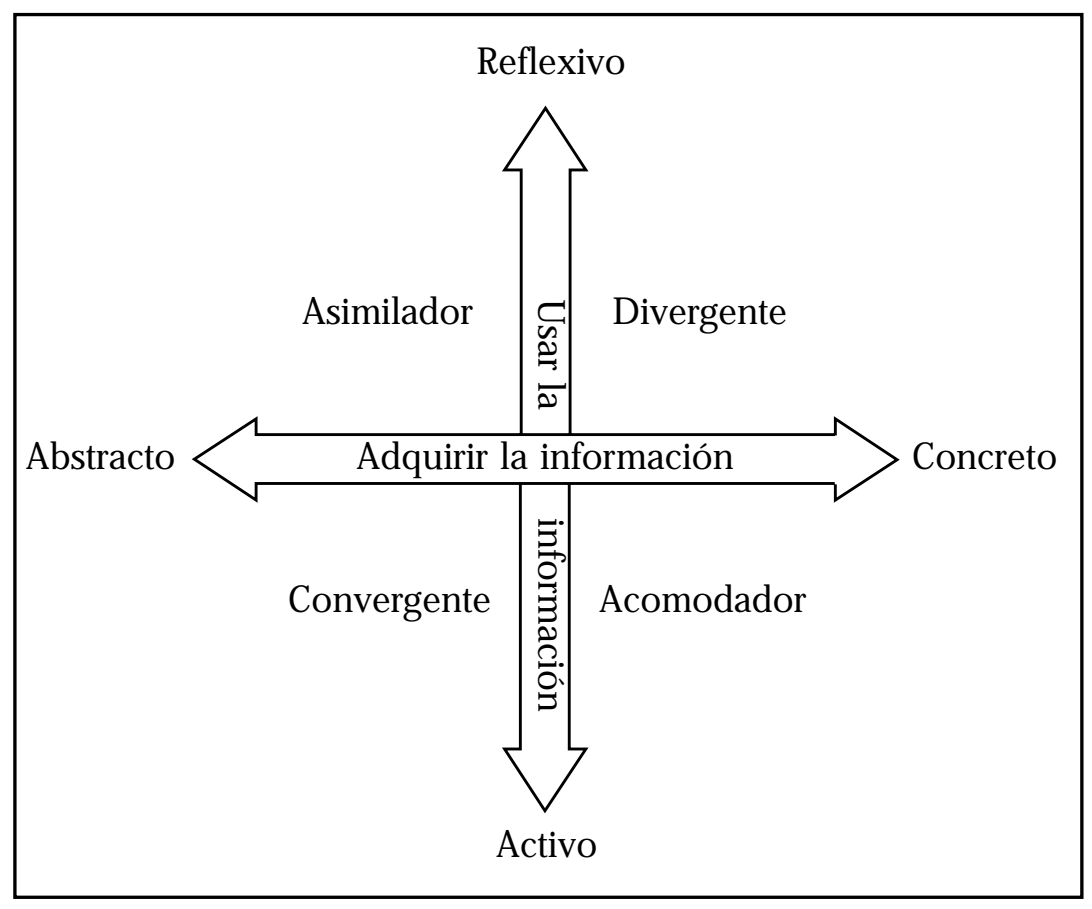


aprende mejor haciendo ${ }^{15}$ (Figura 2). Al clasificar a estos 4 aprendices según su modo de adquirir información, distinguimos dos aprendices abstractos (Asimilador y Convergente) y dos concretos (Divergente y Acomodador). Por otra parte, según su modo de procesar la información, tenemos un par de aprendices reflexivos (Asimilador y Divergente) y otro par activo (Convergente y Acomodador) ${ }^{15}$.

Rendimiento académico. El rendimiento académico se operacionalizó a través de la Calificación Médica Nacional (CMN) y las notas obtenidas durante la carrera, en cada uno de los 3 ciclos: Ciclo Básico ( $1^{o}$ y $3^{\circ}$ ) , Ciclo Preclínico ( $4^{\circ}$ y 5oㅜ), y Ciclo Clínico (6oㅜ y 7oㅜ).

Se escogió la Calificación Médica Nacional por ser un indicador global de rendimiento académico que tiene un impacto importante en las posibilidades profesionales de los egresados. La CMN, desarrollada por la Asociación de Facultades de Medicina de Chile (ASOFAMECH), usa una escala de 1 a 7 y se calcula según la siguiente fórmula:

$$
\mathrm{CMN}=0,6 \mathrm{~N}+0,3 \mathrm{I}+0,1 \mathrm{E}
$$

donde $\mathrm{N}=$ promedio de notas de $1^{0}$ a $5^{0}$ año, $\mathrm{I}=$ nota del examen final de internados y $\mathrm{E}=$ nota de presentación internado de $6 \underline{0}$ año.

Estadística. Se utilizaron las pruebas estadísticas ' $t$ ', Chi cuadrado o ANOVA, según correspondiera.

\section{Resultados}

Preferencias psicológicas. Analizadas las preferencias psicológicas de los 66 estudiantes, observamos que igual porcentaje prefirió Extraversion (E) que Introversion (I) (Tabla 2). Lo mismo ocurrió con la percepción de información (S-N). Sin embargo, hubo una distribución desigual de los alumnos en cuanto al discernimiento [77\% Thinking (T) vs $23 \%$ Feeling (F)] y la postura frente a la vida $[67 \%$ Judging (J) vs 33\% Perceiving (P)] (Tabla 2).

Las preferencias psicológicas de hombres y mujeres fueron diferentes en todas las dimensio-
Tabla 2. Preferencias psicológicas de los estudiantes (\%)

\begin{tabular}{|lccc|}
\hline & $\begin{array}{c}\text { Todos } \\
(\mathrm{n}=66)\end{array}$ & $\begin{array}{c}\text { Hombres } \\
(\mathrm{n}=40)\end{array}$ & $\begin{array}{c}\text { Mujeres } \\
(\mathrm{n}=26)\end{array}$ \\
\hline $\mathrm{E}$ & 52 & 47 & $58^{\mathrm{a}}$ \\
$\mathrm{I}$ & 48 & 53 & $42^{\mathrm{a}}$ \\
$\mathrm{S}$ & 50 & 47 & 54 \\
$\mathrm{~N}$ & 50 & 53 & 46 \\
$\mathrm{~T}$ & $77^{*}$ & 88 & $62^{\mathrm{a}}$ \\
$\mathrm{F}$ & 23 & 12 & $38^{\mathrm{a}}$ \\
$\mathrm{J}$ & $67^{*}$ & 62 & $73^{\mathrm{a}}$ \\
$\mathrm{P}$ & 33 & 38 & $27^{\mathrm{a}}$ \\
\hline
\end{tabular}

${ }^{*}$ p $<0,001$ respecto del polo opuesto de la misma dimensión ( $p$ ej: $T$ vs $F$ ), a $p<0,001$ respecto del valor correspondiente de los hombres.

nes, excepto en la percepción de la información (S-N): una mayor proporción de mujeres tenía preferencias Extraversion (E), Feeling (F) y Judging (J) (Tabla 2).

Estilos de aprendizaje. EI estilo de aprendizaje más frecuente fue el Asimilador (44\%), seguido por el Convergente (35\%), el Divergente (11\%) y, finalmente, el Acomodador (10\%) (Figura 3). Los aprendices abstractos (Asimiladores + Convergentes) concentraron a 79\% de la población y los concretos (Divergentes + Acomodadores) a 21\% restante. Por otra parte, más de la mitad de nuestros estudiantes (55\%) eran aprendices reflexivos (Asimiladores + Divergentes), y el $45 \%$ restante, activos (Convergentes + Acomodadores).

Rendimiento académico. Las notas de la CMN fluctuaron entre 5,60 y 6,66, con un valor promedio

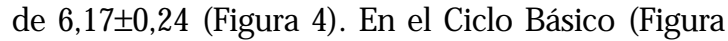
5), las notas siguieron un patrón de distribución aproximadamente normal, con un promedio de 5,55 $\pm 0,40$. En el Ciclo Preclínico, en cambio, la distribución es bimodal. El grupo de menor rendi-

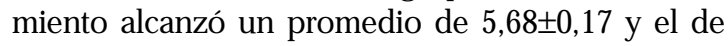
mayor rendimiento un promedio de $6,24 \pm 0,16$. Por

\footnotetext{
aPromedio de las notas de las asignaturas de los años correspondientes, ponderado por el número de créditos respectivo.
} 
último, las notas del Ciclo Clínico alcanzaron un promedio de $6,70 \pm 0,17$ y presentaron una distribución aparentemente normal que se trunca en el extremo superior de la escala (Figura 5).

Preferencias psicológicas y rendimiento académico. $\mathrm{Al}$ analizar los resultados de la $\mathrm{CMN}$ en relación con las preferencias psicológicas observamos diferencias significativas en las dimensiones E-l y T-F (Tabla 3). Los estudiantes con preferencia E tuvieron un promedio levemente mejor que los con preferencia I. Asimismo, los estudiantes con preferencia $\mathrm{F}$ tuvieron mejor promedio que los $\mathrm{T}$ (Tabla 3). Al estratificar por sexo, se pierde la diferencia E-

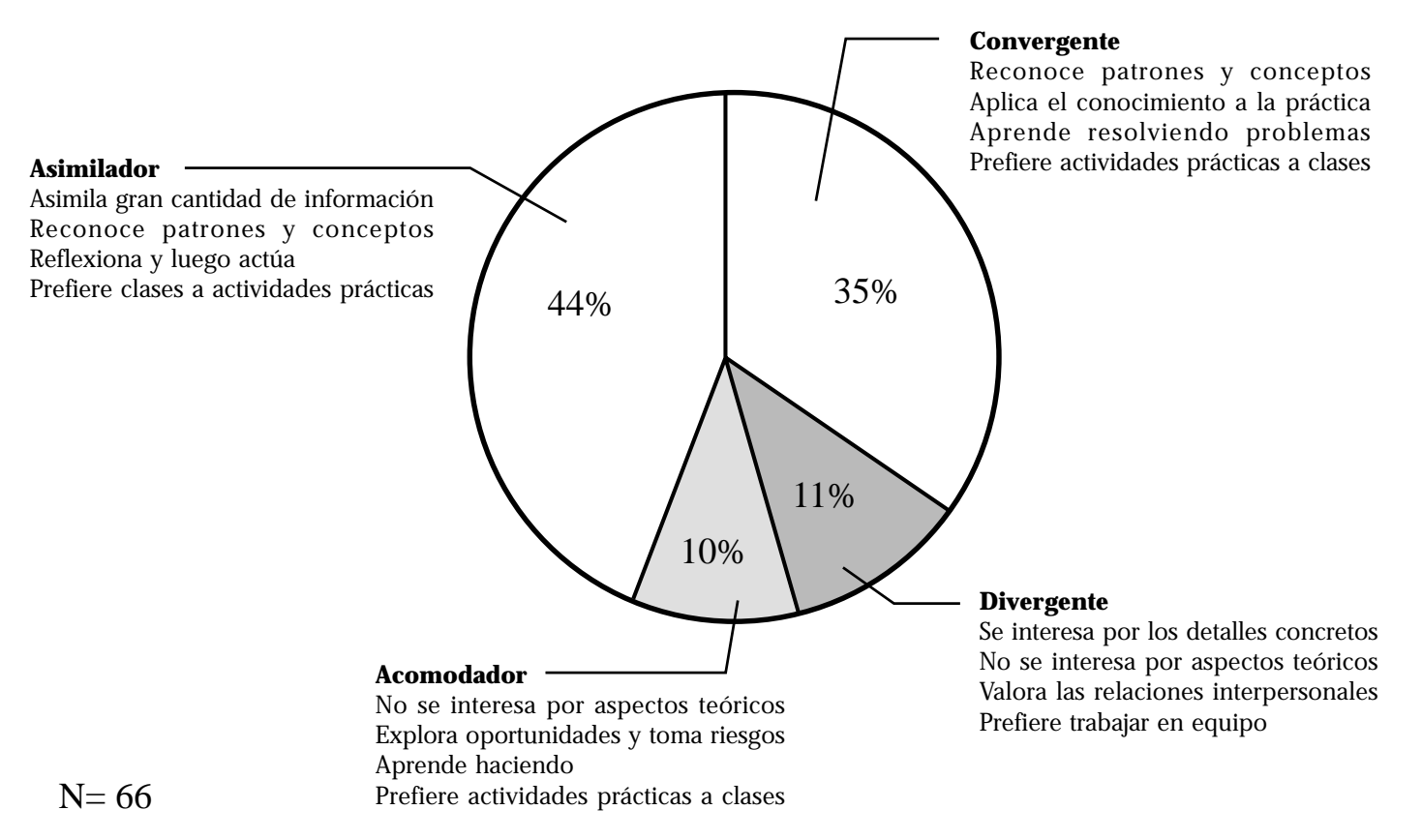

FIGURA 3. Estilos de aprendizaje de los estudiantes.

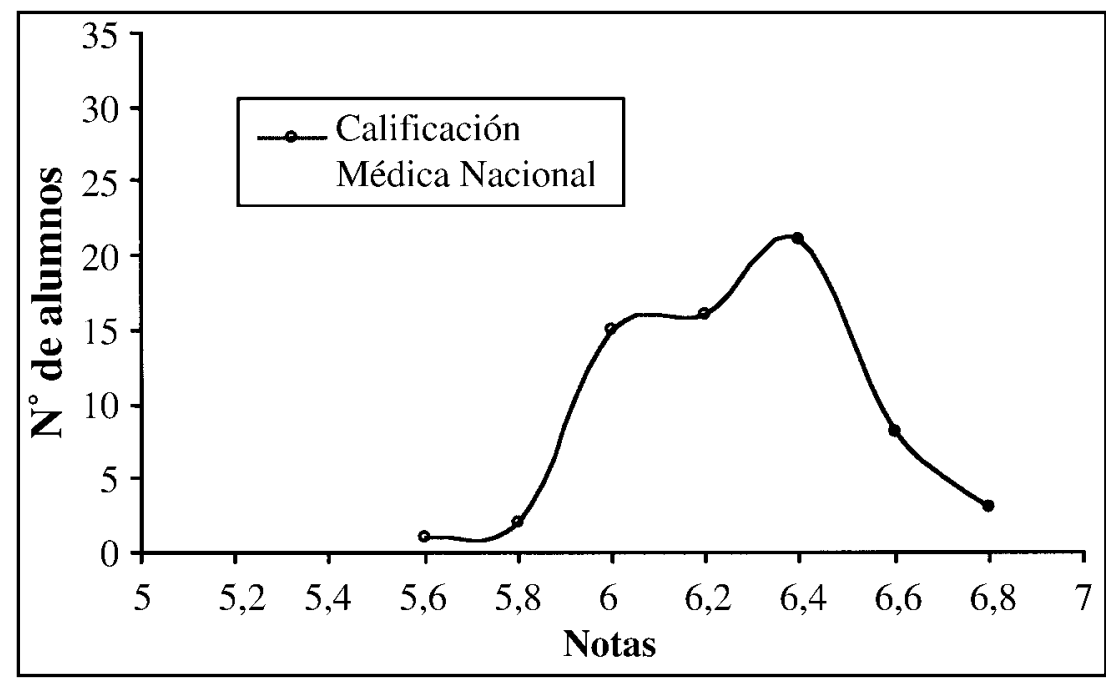

Figura 4. Distribución de la Calificación Médica Nacional. 


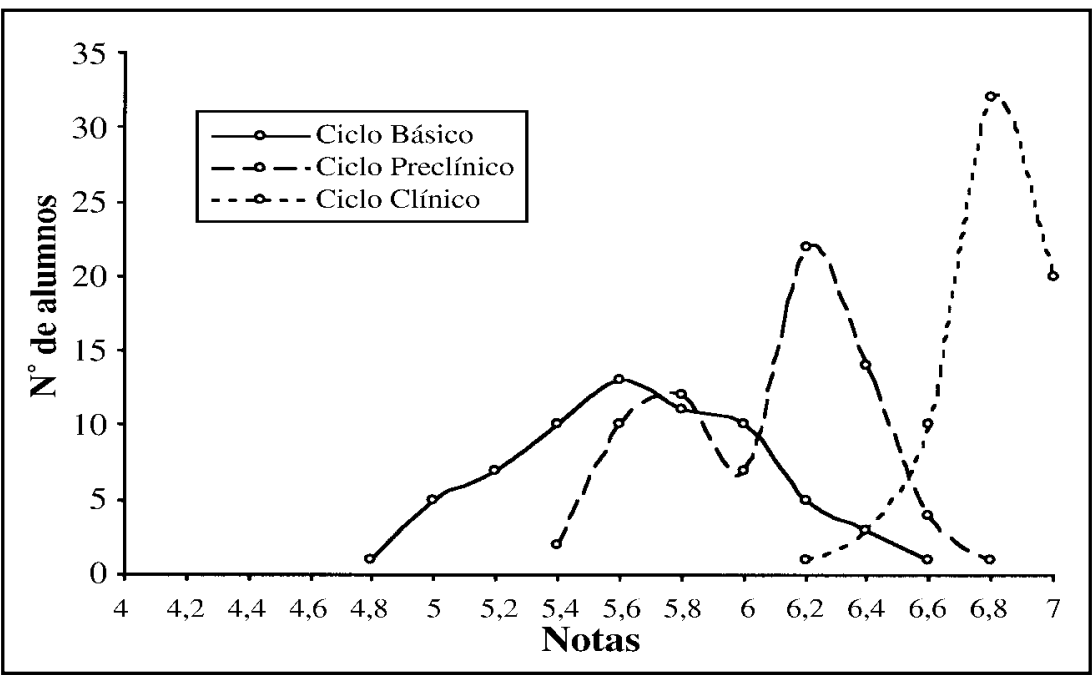

FIgURA 5. Distribución de las notas por ciclo.

Tabla 3. Calificación M édica Nacional y promedio por ciclo según las preferencias psicológicas

\begin{tabular}{|c|c|c|c|c|c|c|c|c|c|c|c|c|}
\hline & \multicolumn{3}{|c|}{$\begin{array}{c}\text { Calificación } \\
\text { Médica Nacional }\end{array}$} & \multicolumn{3}{|c|}{ Básico } & \multicolumn{3}{|c|}{$\begin{array}{c}\text { Ciclos } \\
\text { Preclínico }\end{array}$} & \multicolumn{3}{|c|}{ Clínico } \\
\hline & $\mathrm{T}$ & $\mathrm{H}$ & M & $\mathrm{T}$ & $\mathrm{H}$ & M & $\mathrm{T}$ & $\mathrm{H}$ & M & $\mathrm{T}$ & $\mathrm{H}$ & M \\
\hline E & $6,2 *(0,2)$ & $6,2(0,2)$ & $6,3(0,2)$ & $5,6(0,4)$ & $5,6(0,3)$ & $5,7(0,5)$ & $6,1^{*}(0,3)$ & $6,0(0,3)$ & $6,2(0,3)$ & $6,8^{*}(0,2)$ & $6,7(0,2)$ & $6,8(0,1)$ \\
\hline I & $6,1(0,2)$ & $6,0(0,3)$ & $6,2(0,2)$ & $5,5(0,4)$ & $5,5(0,4)$ & $5,6(0,4)$ & $5,9(0,3)$ & $5,8(0,3)$ & $6,1(0,3)$ & $6,7(0,2)$ & $6,6(0,2)$ & $6,7(0,1)$ \\
\hline S & $6,2(0,2)$ & $6,1(0,3)$ & $6,3(0,2)$ & $5,6(0,4)$ & $5,6(0,4)$ & $5,7(0,4)$ & $6,0(0,4)$ & $5,9(0,4)$ & $6,2(0,3)$ & $6,7(0,2)$ & $6,7(0,2)$ & $6,8(0,1)$ \\
\hline $\mathrm{N}$ & $6,1(0,2)$ & $6,1(0,2)$ & $6,1(0,3$ & $5,5(0,4)$ & $5,5(0,4)$ & $5,5(0,5)$ & $6,0(0,3)$ & $5,9(0,3)$ & $6,1(0,3)$ & $6,7(0,1)$ & $6,7(0,2)$ & $6,7(0,1)$ \\
\hline $\mathrm{T}$ & $6,1(0,2)$ & $6,1(0,2)$ & $6,2(0,2)$ & $5,5(0,4)$ & $5,5(0,4)$ & $5,6(03)$ & $5,9(0,3)$ & $5,9(0,3)$ & $6,1(0,3)$ & $6,7(0,2)$ & $6,7(0,2)$ & $6,7(0,1)$ \\
\hline F & $6,3^{* *}(0,2)$ & $6,4^{*}(0,3)$ & $6,3(0,3)$ & $5,8^{*}(0,5)$ & $5,8(0,4)$ & $5,7(0,6)$ & $6,2 *(0,3)$ & $6,3^{* *}(0,2)$ & $6,2(0,3$ & $6,8^{* *}(0,1)$ & $6,8^{*}(0,1)$ & $6,8(0,1)$ \\
\hline $\mathrm{J}$ & $6,2(0,2)$ & $6,1(0,3)$ & $6,3(0,2)$ & $5,6(0,4)$ & $5,5(0,4)$ & $5,7(0,4)$ & $6,1(0,3)$ & $5,9(0,3)$ & $6,2 *(0,3)$ & $6,7(0,2)$ & $6,7(0,2)$ & $6,8^{*}(0,1)$ \\
\hline $\mathrm{P}$ & $6,1(0,2)$ & $6,1(0,2)$ & $6,1(0,2)$ & $5,5(0,4)$ & $5,5(0,4)$ & $5,4(0,5$ & $5,9(0,3)$ & $5,9(0,3)$ & $5,9(0,3)$ & $6,7(0,2)$ & $6,7(0,2)$ & $6,7(0,1)$ \\
\hline
\end{tabular}

T: todos, H: hombres y M: mujeres. Los resultados se expresan como valor promedio, con la desviación estándar entre paréntesis. **. $\mathrm{p}<0,01 ;{ }^{*} \mathrm{p}=0,05$ respecto del valor del polo opuesto de la misma dimensión ( $\mathrm{p}$ ej: T vs F).

I, en cambio, se mantiene la diferencia T-F, aunque sólo en los hombres. Aquellos hombres con preferencia Feeling $(\mathrm{F})$ obtuvieron $\mathrm{CMN}$ tres décimas más altas que los hombres con preferencia Thinking (T) (Tabla 3). Análogamente, en el Ciclo Básico los estudiantes con preferencia Feeling (F) obtuvieron notas superiores a los Thinking (T) (Tabla 3). Esta diferencia, sin embargo, desapareció al analizar separadamente a hombres y mujeres. En el Ciclo Preclínico también hallamos diferencias significativas en las dimensiones E-I y T-F. Después de estratificar a los estudiantes por sexo, sólo permaneció la diferencia en la dimensión T-F en los hombres, en los que alcanzó 4 décimas. Lo mismo ocurrió en el Ciclo Clínico; de las diferencias observadas sólo se conservó la diferencia en T-F en los hombres (Tabla 3).

A diferencia de lo que ocurrió en los hombres, en las mujeres el rendimiento estuvo asociado a la actitud frente a la vida. Las mujeres sistemáticas y 
estructuradas (J) tuvieron mejor promedio en los ciclos Preclínico y Clínico que las jóvenes más perceptivas y flexibles (P) (Tabla 3).

En síntesis, los hombres introvertidos y lógicos obtuvieron notas inferiores al resto de sus compañeros. Por otra parte, las mujeres sistemáticas y estructuradas obtuvieron mejor promedio que las jóvenes más perceptivas y flexibles. Estas diferencias se manifestaron más claramente a partir del $4^{\circ}$ año de carrera (Ciclo Preclínico).

Pares de preferencias y rendimiento académico. Al comparar las notas obtenidas en el Ciclo Preclínico por los varones que prefirieron simultáneamente Introversion y Thinking (grupo IT), con las de aquellos que no tuvieron ninguna de estas preferencias o sólo una de ellas (grupo no-IT) observamos una segregación notoria (Figura 6). El grupo IT obtuvo notas significativamente menores que el no-IT $(5,74 \pm 0,24$ vs $6,04 \pm 0,29 ; p \varangle 0,001)$. A diferencia de los alumnos IT cuyas notas se distribuyeron normalmente, la distribución de notas de los alumnos no-IT fue bimodal. Interesantemente, el pico de menor rendimiento del grupo no-IT comespondió a los únicos 6 alumnos con preferencias ET (datos no mostrados).

Una situación completamente diferente ocurrió con el rendimiento de las mujeres (Figura 7). La distribución de notas en el Ciclo Preclínico de las alumnas con preferencias IT se superpuso a la de las alumnas con preferencias no-IT.
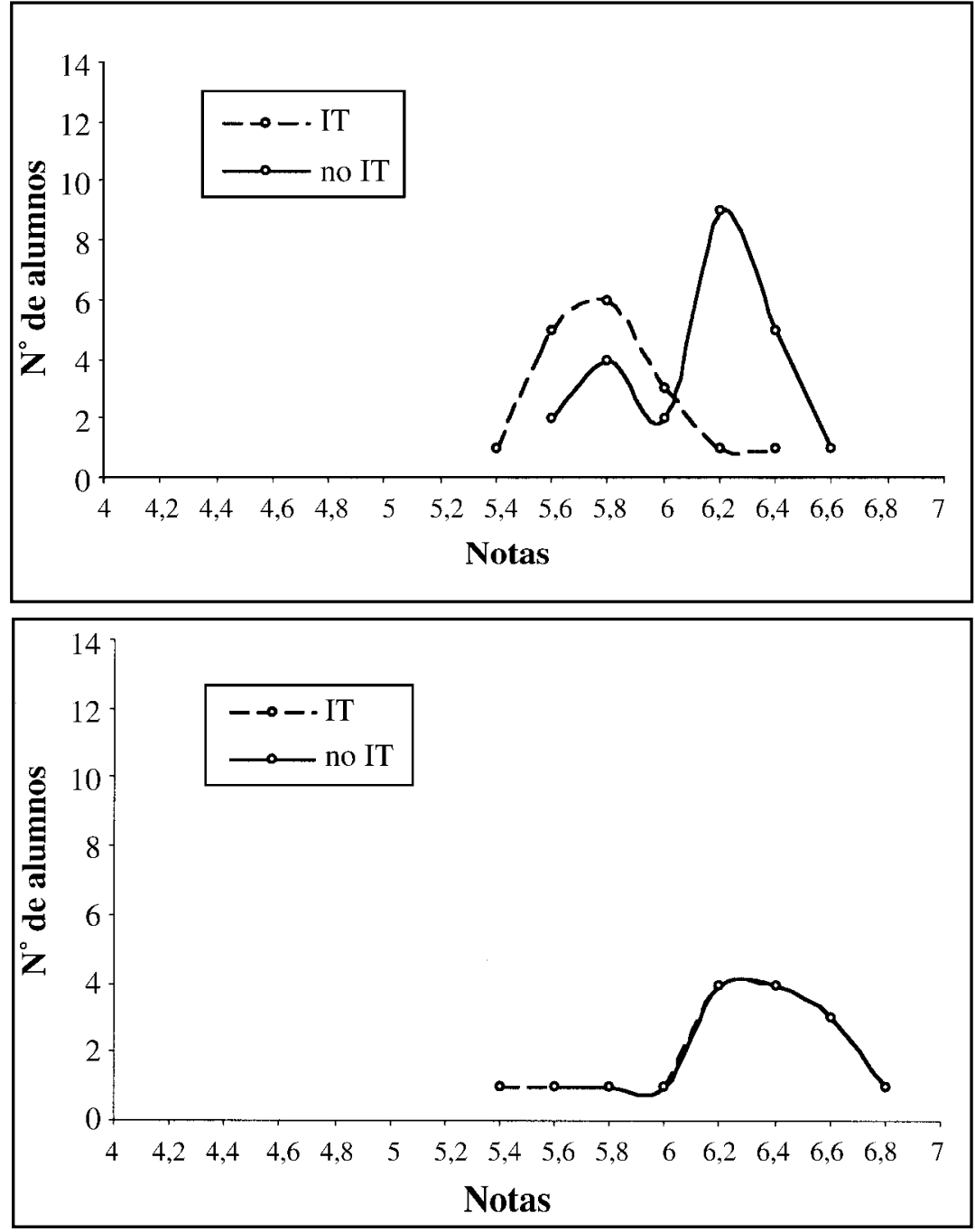

FIgURA 6. Distribución de las notas de los hombres con preferencias IT y no-IT en el Ciclo Preclínico.
Figura 7. Distribución de las notas de las mujeres con preferencias IT y no-IT en el Ciclo Preclínico. 
Estilos de aprendizaje y rendimiento académico. El rendimiento académico de los alumnos no estuvo relacionado con su estilo de aprendizaje (datos no mostrados).

\section{DisCUSIÓN}

Preferencias psicológicas y rendimiento académico. El rendimiento académico de los estudiantes de medicina de esta cohorte está relacionado con sus preferencias psicológicas. Estas asociaciones se hacen notorias a partir del Ciclo Preclínico y, algunas de ellas, se manifiestan también en la CMN. En los hombres, las dimensiones involucradas son la fuente de motivación y el discernimiento, y en las mujeres la actitud frente a la vida. Los hombres introvertidos y lógicos obtienen notas inferiores al resto de sus compañeros. Por otra parte, las mujeres sistemáticas y estructuradas tienen mejor promedio que las jóvenes más perceptivas y flexibles. En general, en los hombres las diferencias son de mayor magnitud y trascendencia que en las mujeres.

El hecho que las diferencias se hagan evidentes a partir del Ciclo Preclínico, sugiere que el aprendizaje en contacto con los pacientes, con sus formas particulares de adiestramiento y evaluación, constituye un desafío diferente para los alumnos, según sean sus características psicológi$\mathrm{cas}^{8}$. Otra diferencia importante entre el Ciclo Básico y Preclínico es que en este último se ponen en juego habilidades que no eran tan relevantes en los años previos, como la comunicación interpersonal y la capacidad para integrar el conocimiento. Por otra parte, las evaluaciones se tornan más subjetivas y prácticas e introducen -además de elementos cognitivos- aspectos afectivos y psicomotores, cuyo desarrollo varía según las características psicológicas del estudiante. Parece comprensible entonces, que a los estudiantes con preferencias IT, especialmente dotados para la introspección y el análisis lógico, les sea más difícil sobresalir en contextos que requieren habilidades que les son menos propias, como la capacidad de comunicarse en forma fluida y empática con los pacientes ${ }^{12,22,23}$.

La falta de asociación entre las preferencias IT y el rendimiento, en las mujeres, puede atribuirse a influencias culturales, que asocian ser mujer con una mayor capacidad de comunicarse ${ }^{24}$. Por ello, aun cuando la mujer tenga preferencias IT, es posible que sus habilidades interpersonales estén suficientemente desarrolladas como para desempeñarse en forma satisfactoria en el ámbito interpersonal. Análogamente, la falta de asociación entre la preferencia Judging y el desempeño académico, en los hombres, podría deberse a la presión social que asocia el ser hombre con tener una postura decidida y estructurada frente a la vida.

La información publicada en relación a preferencias psicológicas y rendimiento de estudiantes y residentes de medicina es muy variada. Algunos estudios evalúan el rendimiento de alumnos en asignaturas básicas del currículo, mientras que otros miden el desempeño de residentes en pruebas generales de competencia clínica. Por ejemplo, se ha reportado que los estudiantes con preferencias Introversion y Thinking (IT) tienen mejor rendimiento en Fisiología ${ }^{25}$. Por otra parte, el mejor rendimiento de residentes de pediatría (medido con el «American Board of Paediatrics In-training Examination») está asociado a la preferencia por Extroversion (E) y de Medicina Familiar (medido con el «n-Service Training Examination» a las preferencias Feeling y Judging $^{26,27}$.

A pesar de la diversidad metodológica de los estudios, éstos parecen tener algunas consistencias básicas con nuestros resultados. Mientras que nosotros encontramos una asociación negativa entre las preferencias Introversion y Thinking (IT) y el rendimiento en el ciclo preclínico y clínico, los estudios mencionados reportan una asociación positiva entre el desempeño clínico y las preferencias opuestas (ie: Extraversion y Feeling).

Estilos de aprendizaje y rendimiento académico. A diferencia de lo reportado en otros estudios ${ }^{28-31}$, nosotros no encontramos asociación entre los estilos de aprendizaje y el rendimiento académico. Sin embargo, existen diferencias importantes de diseño: los reportes publicados, normalmente se refieren a notas de una asignatura y no a promedios de ciclos o notas globales de la carrera, como nuestro estudio. Es posible que al utilizar promedios de asignaturas con metodologías instruccionales y formas de evaluación distintas, se hayan diluido o perdido las relaciones con los estilos de aprendizaje. 
Conclusiones generales y limitaciones. El rendimiento académico de los estudiantes de medicina está relacionado en forma conjunta con las preferencias psicológicas y el sexo. En los hombres, el interés por los demás y la capacidad de tomar decisiones que favorezcan la armonía entre las personas están asociados con un mejor desempeño. En las mujeres, en cambio, es la tendencia a funcionar de manera sistemática y estructurada la característica que se relaciona con mejor rendimiento.

Pensamos que nuestros hallazgos son relevantes para el futuro profesional de nuestros egresados, pues diferencias de 3 a 4 décimas en la CMN, como las encontradas aquí, pueden determinar el éxito o fracaso en su postulación a una beca de especialización o un cargo.

Aunque, a primera vista, llama la atención que el rendimiento se correlacione con las características de personalidad y no con los estilos de aprendizaje de los alumnos, es importante considerar que, en este estudio, el rendimiento se operacionalizó a través de indicadores globales de rendimiento de ciclos completos o de toda la carrera, que abarcan años de desempeño en múltiples asignaturas y experiencias de aprendizaje con variadas metodologías ${ }^{32}$. Parece razonable pensar que estas medidas globales se correlacionen más con rasgos profundos y estables, como son las preferencias psicológicas, que con los estilos de aprendizaje, que están asociados a situaciones más específicas de aprendizaje.

Reconocemos que la principal limitación de este estudio deriva de su carácter retrospectivo, y que una respuesta más definitiva a la pregunta que nos preocupa sólo podrá obtenerse del estudio prospectivo en curso (Psimed 21). Sin embargo, dado que en estudios test-retest hemos encontrado que las preferencias psicológicas y estilos de aprendizaje de los estudiantes de medi-

\section{REFERENCIAS}

1. Newble D, Entwistle N. Learning Styles and approaches: implications for medical education. Med Educ 1986; 20: 162-75.

2. Lester W, JuschKa B, Mandin H. Assessing the Psychological Types of specialists to assist students in career choice. Acad Med 1995; 70: 932-3. cina son bastante estables en el tiempo (82\% de mantención a los 2 años, datos no mostrados), hemos considerado útil realizar este estudio. En el mismo sentido, la semejanza entre la cohorte de egreso 2001 (motivo de este estudio) y las cohortes de ingreso 2000-2002 ${ }^{11}$, en cuanto a preferencias psicológicas y estilos de aprendizaje apoya la idea que estas variables no se modificarían considerablemente durante la carrera.

Respecto de la generalizabilidad de nuestros hallazgos, no estamos en condiciones de establecer si las asociaciones aquí encontradas entre las preferencias psicológicas y el rendimiento académico son propias de la cohorte de egreso 2001 o comunes a los estudiantes de medicina de nuestra escuela, o de las escuelas de medicina chilenas en general. Sin embargo, nos atrevemos a especular que son generalizables, al menos, a los estudiantes de medicina de nuestra escuela, ya que la cohorte de este estudio (egreso 2001) tiene características biodemográficas, académicas y psicológicas semejantes a las cohortes de ingreso reciente ${ }^{11}$.

Tenemos conciencia que el rendimiento académico es resultante de múltiples factores, entre los cuales se hayan las preferencias psicológicas y que estamos tratando con jóvenes en formación, lo que hace aconsejable una cautela especial en la extrapolación de los resultados.

En conclusión, este estudio sugiere que las preferencias psicológicas pueden incidir en el rendimiento académico de los estudiantes de medicina, particularmente en los ciclos preclínico y clínico. En este sentido, nos parece importante que los docentes y autoridades involucradas en la formación de nuevos médicos conozcan a sus alumnos desde la perspectiva de sus preferencias psicológicas. Este conocimiento puede iluminar el diseño de actividades que potencien el desarrollo de habilidades no cognitivas que hoy facilitarán el aprendizaje y mañana el desempeño profesional de sus egresados.

3. McGrath E, Zimet N. Female and male medical students: differences in specialty choice selection and personality. J Med Educ 1977; 52: 293-300.

4. SHEN H, Comrey L Predicting medical students academic performances by their cognitive abilities and personality characteristics. Acad Med 1997; 72: 781-6.

5. TutTon P. Psychometric tests results associated with high achievement in basic science compo- 
nents of a medical curriculum. Acad Med 1996; 72: 181-6.

6. WaLCK M, Cambre K, RandaL H. Personality type and medical specialty. J La State Med. Soc 1999; 151: 463-9.

7. Peng R, Khaw $H$, Edariah A. Personality and performance of preclinical medical students. Med Educ 1995; 29: 283-8.

8. CuRRY L. Cognitive and learning styles in medical education. Acad Med 1999; 74: 409-13.

9. Rissetti F, Himmel E, González J, Galardo G. Relación entre variables de personalidad y adaptación y rendimiento universitario: un estudio longitudinal. Bol Inv Educ Fac Educ Stgo 1996; 11: 11-29.

10. RIsSETTI F. Variables motivacionales y de personalidad en la admisión a la Escuela de Medicina. Rev Méd Chile 1997; 125: 45-62.

11. Bitran M, Zúñiga D, Lafuente M, Viviani P, Mena B. Tipos psicológicos y estilos de aprendizaje de los estudiantes que ingresan a Medicina en la Pontificia Universidad Católica de Chile. Rev Méd Chile 2003; 131: 1067-78.

12. Briggs Myers I. MBTI: Inventario Tipológico Forma G. Manual. Madrid: TEA Ediciones. 1991.

13. Myers I, MacCauley M, Quenk N, Hammer A. MBTI Manual: A guide to the development and use of the Myers-Briggs Type Indicator. Palo Alto, California: Consulting Psychologist Press, Inc. 1998.

14. Коцв D. Inventario de los estilos de aprendizaje: Inventario autoevaluativo y su interpretación. Training Resources Groups, Hay/McBer. Boston, 1981.

15. Koьв D. Experiential Learning: Experience as the Source of Learning and Development. New Jersey: Prentice Hall, PTR. 1984.

16. InCLAN A. The development of the Spanish version of the Myers Briggs Type Indicator, Form G. Journal of Psychological Type 1986; 11: 35-46.

17. Capraro R, Capraro M. Myers-Brigg Type Indicator: score realiability across studies: a metaanalytic reliability generalization study. Educational and Psychological Measurement 2002; 62: 590-602.
18. Wawck M, Cambre K. Personality Types in Academic Medicine. J La State Med Soc 1999; 151: 378-83.

19. Stilwell N. WaLick M, Thal S, Burleson J. MyersBriggs type and medical specialty choice: a new look at an old question. Teach Learn Med 2000; 12: 14-20.

20. Ciack G, Head J. The personality 'types' of medical students and the implications for medical education. Medical Teacher 1998; 20: 57.

21. Jung C. Psychological Types. Routledge and Megan. Londres. 1971.

22. Myers I, Myers P. Gifts differing: understanding personality type. Palo Alto. California: Davies Black Publishing, 1995.

23. Aluen J, Brock S. Health care communication using personality type. Patients are different. Routledge, Londres, 2000.

24. Clack G, Head J. Gender differences in medical graduates' assessment of their personal attributes. Med Educ 1999; 33: 101-5.

25. Tharp G. Relationship between personality type and achievement in an undergraduate physiology course. Am J Physiol 1992; 262: S1-3.

26. LACORTE M. Personality, clinical performance and knowledge in pediatric residents. Med Educ 1993; 27: 165-9.

27. BIAKE G. Resident's formal knowledge acquisition and preferred leaming styles. Fam Med 1995; 27: 35-8.

28. Lynch T, Wowlfl N, Steele D, Hanssen D. Learning Style influences student examination performance. Am J Surg 1998; 176: 62-6.

29. Newland J, Woelfl. Learning style and academic performance within a group of sophomore Medical students. Acad Med 1992; 67: 349.

30. Davies S, Rutledge C, Davies T. Students' learning styles do affect performance. Acad Med 1995; 70: 659-60.

31. SOBRAL D. Diagnostic ability of medical students in relation to their learning characteristics and preclinical background. Med Educ 1995; 29: 278-82.

32. Bitran M, Wright A, Zúñiga D, Mena B, Velasco N, Moreno R. Mejoría en el desempeño académico de estudiantes de medicina en tiempos de reforma curricular. Rev Méd Chile 2002; 130: 437-45. 